\title{
Tecnologias Educacionais acessíveis para apoiar o Ensino de Matemática: Uma Revisão Sistemática de Literatura
}

\author{
Miriam Garcia Müller- PPGIE - UFRGS - miriam.muller@ canoasedu.rs.gov.br- \\ https://orcid.org/0000-0002-9885-4578 \\ Crediné Silva de Menezes - PPGIE - UFRGS - RS - Brazil - credine@ gmail.com ' \\ https://orcid.org/0000-0002-2709-7135
}

Resumo. Apresentamos uma Revisão Sistemática de Literatura (RSL) sobre o uso de Arquiteturas Pedagógicas (A.P) e tecnologias para apoiar o ensino de matemática a educandos com Transtorno do Espectro Autista e Deficiência Intelectual. A metodologia se baseou no protocolo de RSL consolidado por Kitchenham (2004). Utilizou-se como corpus de informação os periódicos científicos que atendiam aos critérios de inclusão e exclusão definidos no protocolo de pesquisa. Foram encontrados 361 trabalhos, desses, 21 foram selecionados e analisados, de forma a responder as questões de perguntas estabelecidas. Conclui-se que ainda é incipiente o número de pesquisas sobre o uso de tecnologias para apoiar a construção de conhecimentos matemáticos para sujeitos com TEA e/ou Deficiência Intelectual. Ao tratarmos especificamente do ensino de álgebra mediados por tecnologias os resultados foram mais escassos, sendo localizado apenas um trabalho nesta área de ensino e os demais tratava-se das quatro operações matemáticas.

Palavras-chaves: Arquiteturas Pedagógicas; tecnologias; matemática; álgebra; Transtorno do Espectro Autista e/ou deficiência intelectual.

\section{Accessible Educational Technologies to Support Mathematics Teaching: A Systematic Literature Review}

\section{Abstract.}

We presente a Systematic Literature Review (RSL) on the use of Pedagogical Architectures (A.P) and technologies to support the teaching of mathematics for students with Autistic Spectrum Disorder and Intellectual Disability. The methodology was based on the RSL protocol consolidated by Kitchenham (2004). Scientific journals that met the inclusion and exclusion criteria of the research protocol were used as a corpus of information. A total of 361 works were selected, proposals, 21 were selected and completed, in order to answer the questions of the survey. It is concluded that the number of researches on the use of technologies to support the construction of mathematical knowledge for subjects with ASD and/or Intellectual Disability is still incipient. When dealing specifically with the teaching of algebra mediated by technologies, the results were scarcer, with only one work being located in this teaching area and the others dealing with four mathematical operations.

Keywords: Pedagogical Architectures; technologies; algebra; math; Autistic Spectrum Disorder and/or intellectual disability.

\section{Introdução}

A alfabetização matemática é a base para que os sujeitos relacionem conhecimentos necessários para entender o mundo e ter autonomia frente a situações do dia-a-dia. Porém, ensinar matemática (é) tem se tornado um desafio maior frente às novas tecnologias, pois muitos estudantes consideram uma das disciplinas "difíceis", a qual, muitos deles, "têm 
medo". Possivelmente, esses "medos" ocorram pelo fato de, durante o processo de escolarização, terem sidos submetidos a métodos de ensinos engessados. Assim, os professores, na tentativa de "descomplicar" os conceitos, buscam pensar nas representações e nos recursos para auxiliar nos processos de aprendizagem, com abordagens problematizadoras, mas que coloque o sujeito como protagonista na sua aprendizagem.

Dentre as dificuldades apresentadas no ensino de matemática, uma das subáreas mais problemáticas para os educandos é a Álgebra, por trabalhar habilidades cognitivas que desenvolvem um tipo de raciocínio específico, o pensamento algébrico, envolvendo generalizações, simbolismo, relações entre grandezas e padrões e regularidades (DIAS, 2017; PONTE et.al., 2009).

Ao referirmos a inclusão de alunos com Deficiência Intelectual (D.I) ou Transtorno do Espectro Autista (TEA), o desenvolvimento do pensamento algébrico torna-se um desafio maior. As pesquisas apontam que sujeitos com TEA apresentam dificuldades relacionadas a assimilar, abstrair e compreender conceitos abstratos e hipotéticos (DIAS, 2017; BOSA, 2002), bem como os sujeitos com D.I apresentam uma viscosidade genética (oscilações), o que contribui para que tenham um tempo diferenciado na aquisição de novos conceitos, ou seja, podendo levar/dispor de momentos mais longos em cada estádio do desenvolvimento (INHELDER, 1963).

Com o avanço do uso das tecnologias nos ambientes educacionais, estas ferramentas tornaram-se aliadas para apoiar práticas pedagógicas que auxiliem os educandos trabalharem autonomamente em computadores e smartphones, inclusive em atividades assíncronas, de acordo com seu próprio ritmo, com abordagem pedagógica multissensorial, como visual, sonora e interativa, visto que muitos sujeitos são aprendizes visuais. Chen (2012) salienta que o uso das tecnologias pode melhorar a capacidade de atenção, o comportamento social, as interações com pares, capacidade de resposta e desempenho dos sujeitos.

Práticas pedagógicas mediadas pelas tecnologias apresentam-se como uma opção eficaz de oportunizar aprendizagem inovadoras para educandos D.I e TEA e de desenvolver ambientes criativos, personalizados e construtivos, que auxiliam desenvolver habilidades diferenciadas (BURTON et al., 2013), visto que, estes sujeitos costumam responder bem a atividades elaboradas com suporte visual. Os ambientes virtuais permitem exibições visuais dinâmicas e personalizadas, que podem ser adaptadas de acordo com as especificidades e necessidades de cada educando, selecionando os níveis de dificuldade apropriados de acordo as características individuais de cada sujeito.

Nesse contexto, a proposta de Arquiteturas Pedagógicas (A.P), apresentada por Menezes et.al (2013), articula o uso das tecnologias com uma visão pedagógica, e a partir de uma visão ecossistêmica, são definidas como "suportes estruturantes" para apoiar as aprendizagens. Uma A.P é composta pelas combinações de elementos como: abordagem pedagógica, software, internet, inteligência artificial, Educação a Distância, concepção de interconexão em tempos e espaços múltiplos (NEVADO et al., 2011). E, segundo Aragon (2016), o uso de A.P vem possibilitando que os sujeitos desenvolvam a criatividade, autonomia e a cooperação dentro de um microecossistema cognitivo.

Frente a esse contexto, e, também frente às necessidades ocorridas em pesquisa de doutoramento, em que se propõe a criação de uma A.P acessível para apoiar o ensino de álgebra, este artigo apresenta uma investigação aos moldes de levantamento de estado de arte, por meio de Revisão Sistemática de Literatura (RSL), acerca de "artigos científicos" de quais as tecnologias e quais abordagens metodológicas vêm sendo utilizadas para apoiar 
o ensino de matemática a educandos com TEA e/ou D.I. Como objetivo secundário, pretende-se verificar a existência de trabalhos que abordam o uso de A.P ou tecnologias para apoiar especificamente o ensino de Álgebra.

Esta pesquisa segue organizada em quatro seções, a partir desta introdução. A seção 2 discorre sobre os procedimentos metodológicos utilizados na RSL. A seção 3 apresenta os resultados e discussões acerca das questões de pesquisa. $\mathrm{Na}$ seção 4, expõem-se as considerações finais do trabalho.

\section{Metodologia}

Esta pesquisa foi desenvolvida nos moldes indicados por Kitchenham (2004); Kitchenham e Charters (2007) para uma RSL, e foram estabelecidas as três etapas de uma RSL: planejamento, condução e produção do relatório. Dessa forma, o primeiro movimento foi definir o planejamento com a definição das Q.P e dos critérios de inclusão e de exclusão, a condução das buscas foi realizada seguindo as estratégias de buscas e as strings definidas, e por último, realizado a análise que resultou em um relatório de pesquisa. Buscou-se identificar, avaliar e categorizar as produções científicas disponíveis em português, inglês e espanhol referente ao uso de A.P ou tecnologias digitais utilizadas no ensino de matemática para educandos com TEA e D.I.

Após definir o objetivo, foram organizadas as questões de perguntas (QP), que posteriormente deram origem aos termos de buscas.

\subsection{Objetivo e Questões de Pesquisa}

Esta RSL tem como objetivo central encontrar e listar quais arquiteturas pedagógicas ou tecnologias vêm sendo utilizadas para apoiar o ensino de matemática para educandos com TEA e/ou D.I.

A seguir são apresentadas as questões de pesquisa (QP) que nortearam essa RSL:

- QP1: Quais as A.P ou tecnologias que estão sendo usadas para apoiar o ensino de matemática a educandos com TEA e/ou D.I?

- QP2: Existem pesquisas sobre o uso de A.P ou tecnologias para apoiar o ensino específico de Álgebra a educandos com TEA e/ou D.I?

- QP3: O uso de tecnologias digitais no ensino de matemática com educandos com TEA e/ou D.I é realizado de maneira cooperativa entre os sujeitos?

- QP4: As propostas pedagógicas no ensino de matemática com o uso de A.P ou tecnologias digitais para educandos com TEA e/ou D.I baseiam-se em uma abordagem construtivista?

\subsection{Estratégias de Busca e Definição das String}

A partir das questões de pesquisas foram definidos os termos de busca. Essas palavras foram agrupadas formando uma string de busca para cada banco de dados. Foram utilizados os operadores lógicos AND e OR, a fim de combinar as palavras-chaves e verificar o retorno dos trabalhos referentes a este estudo, utilizou-se o operador NOT para excluir termos que não se enquadram no escopo de pesquisa.

As buscas se deram no dia 18 de outubro de 2021 nos seguintes bancos de dados: Portal de Periódicos CAPES/MEC, SciELO - Scientific Electronic Library Online, Scopus ${ }^{\circledR}$, Web of Science ${ }^{\mathrm{TM}}$. $\cdot$ Na tabela 1 é apresentada a string de busca que foi utilizada em cada banco de dados: 


\section{Tabela 1. Strings de busca utilizadas}

\begin{tabular}{|l|l|}
\hline \multicolumn{1}{|c|}{ Banco de dados } & \multicolumn{1}{c|}{ Query (chave) } \\
\hline Periódicos CAPES & $\begin{array}{l}\text { ("pedagogical architecture" OR "learning framework" OR (digital technologies) OR } \\
\text { technologies) AND ("mathematics learning" OR (algebraic reasoning) OR algebraic OR } \\
\text { (algebra learning)) AND ((autism spectrum disorders) OR "intellectual disability" OR } \\
\text { "mental disability") NOT ("machine learning" OR "magnetic resonance imaging") } \\
\text { Refinado por:tópico: Life Sciences \& Biomedicine (remover) coleção: Medline Complete } \\
\text { (remover) } \\
\text { ((pedagogical architecture) OR (learning framework) OR (digital technologies) OR } \\
\text { (technologies)) AND ((mathematics learning) OR (algebraic reasoning) OR (algebraic) OR } \\
\text { (algebra learning)) AND ((autism spectrum disorders) OR (intellectual disability) OR } \\
\text { (mental disability)) }\end{array}$ \\
\hline Web of Science & $\begin{array}{l}\text { ((pedagogical architecture) OR (learning framework) OR (digital technologies) OR } \\
\text { (technologies)) AND ((mathematics learning) OR (algebraic reasoning) OR (algebraic) OR } \\
\text { (algebra learning)) AND ((autism spectrum disorders) OR (intellectual disability) OR } \\
\text { (mental disability)) }\end{array}$ \\
\hline $\begin{array}{l}\text { ((pedagogical architecture) OR (learning framework) OR (digital technologies) OR } \\
\text { (technologies) AND ((mathematics learning) OR (algebraic reasoning) OR (algebraic) OR } \\
\text { (algebra learning)) AND ((autism spectrum disorders) OR (intellectual disability) OR } \\
\text { (mental disability)) }\end{array}$ \\
\hline
\end{tabular}

\subsection{Critérios de Seleção dos Artigos}

Para definir os artigos que seriam selecionados para análise, foi levado em consideração o objetivo deste trabalho e as QP. Foram definidos Critérios de Inclusão e Critérios de Exclusão, conforme apresentado na tabela 2. Estes critérios foram aplicados na análise do título, resumo e palavras-chaves de cada trabalho.

Tabela 2: Critérios de Inclusão e Critérios de Exclusão

\begin{tabular}{|l|l|}
\hline \multicolumn{1}{|c|}{ Critérios de Inclusão } & \multicolumn{1}{c|}{ Critérios de Exclusão } \\
\hline Artigos Completos & $\begin{array}{l}\text { Tão constar as 3 áreas de descritores no título, ou nas } \\
\text { palavras-chaves ou no resumo }\end{array}$ \\
\hline Português, inglês ou espanhol & $\begin{array}{l}\text { Após a leitura do título, palavras-chaves e resumo } \\
\text { identificar que não se trata de trabalhos relacionados } \\
\text { ao objetivo da pesquisa }\end{array}$ \\
\hline Disponíveis para Downloand de forma gratuita & $\begin{array}{l}\text { Artigos Duplicados } \\
\text { Artigos que tenham apenas o título e abstract } \\
\text { disponível }\end{array}$ \\
\hline & $\begin{array}{l}\text { Artigos que tenham mais de uma versão do mesmo } \\
\text { trabalho. }\end{array}$ \\
\hline
\end{tabular}

\subsection{Obtenção dos Artigos e Trabalhos Selecionados:}

A Figura 1 ilustra a sistematização do processo de busca e seleção dos trabalhos, utilizando as strings de buscas definidas na seção 2.2. A pesquisa nos bancos de dados resultou um total de 361 publicações, com 39 artigos excluídos por serem duplicados. Houve, então, a aplicação dos critérios de inclusão e exclusão nos 322 artigos, e a exclusão de 301 após a filtragem. Sobraram 21 artigos que se relacionavam ao uso de tecnologias aplicadas no ensino de matemática a educandos com TEA e/ou D.I. Esses trabalhos foram analisados para a extração dos dados para responder as questões de pesquisa. 


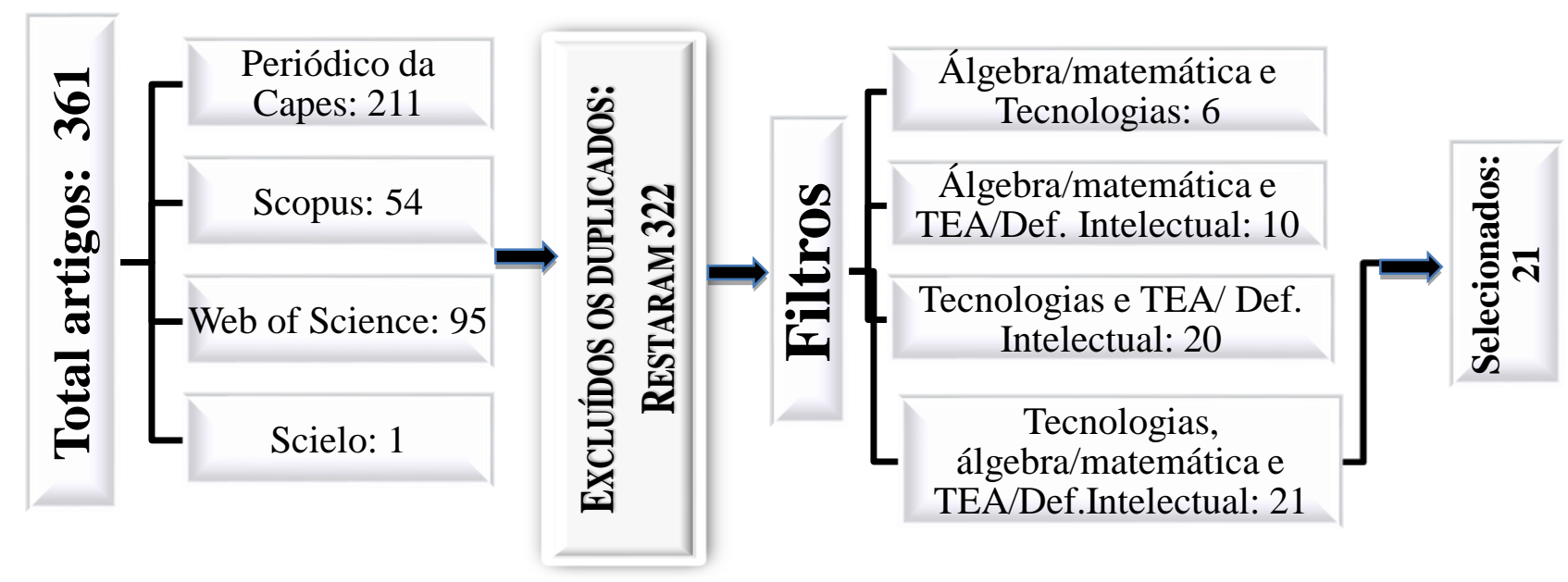

Figura 1. Sistematização das buscas

A tabela 3 exibe os 21 trabalhos selecionados, organizados por ano de publicação, autor e com um identificador (ID) que será utilizado nas análises abordadas na próxima seção.

\section{Tabela 3. Artigos selecionados}

\begin{tabular}{|c|c|c|c|}
\hline Id & Autor & Ano & Título \\
\hline 1 & Reis, M. G. A. D et.al. & 2010 & $\begin{array}{l}\text { Using Information Technology Based Exercises in Primary } \\
\text { Mathematics Teaching of Children with Cerebral Palsy and Mental } \\
\text { Retardation: A Case Study }\end{array}$ \\
\hline 2 & Sula, A. et.al. & 2014 & $\begin{array}{l}\text { A Proposed framework for combining smart environment and heuristic } \\
\text { diagnostic teaching principles in order to assess students' abilities in } \\
\text { math and supporting them during learning. }\end{array}$ \\
\hline 3 & Santos, M.I et.al. & 2015 & $\begin{array}{l}\text { Brief Report: Preliminary Proposal of a Conceptual Model of a Digital } \\
\text { Environment for Developing Mathematical Reasoning in Students with } \\
\text { Autism Spectrum Disorders }\end{array}$ \\
\hline 4 & Zhang, Meilan et.al. & 2015 & $\begin{array}{l}\text { Using Math Apps for Improving Student Learning: An Exploratory } \\
\text { Study in an Inclusive Fourth Grade Classroom }\end{array}$ \\
\hline 5 & Munoz-Soto, R et.al. & 2016 & $\begin{array}{l}\text { Proyect@ Matematicas: A Learning Object for Supporting the } \\
\text { Practitioners in Autism Spectrum Disorders }\end{array}$ \\
\hline 6 & Bakker, Marjoke et.al & 2016 & $\begin{array}{l}\text { Effects of mathematics computer games on special education students' } \\
\text { multiplicative reasoning ability }\end{array}$ \\
\hline 7 & Santos, Maria Isabel et.al. & 2017 & $\begin{array}{l}\text { Design approach of mathematics learning activities in a digital } \\
\text { environment for children with autism spectrum disorders }\end{array}$ \\
\hline 8 & Spooner, F et.al. & 2017 & $\begin{array}{l}\text { Promoting Access to Common Core Mathematics for Students with } \\
\text { Severe Disabilities Through Mathematical Problem Solving }\end{array}$ \\
\hline 9 & Root, Jenny R et.al. & 2017 & $\begin{array}{l}\text { Schema-Based Instruction With Concrete and Virtual Manipulatives to } \\
\text { Teach Problem Solving to Students With Autism }\end{array}$ \\
\hline 10 & Bouck, E.C. et.al. & 2018 & $\begin{array}{l}\text { Adding It Up: Comparing Concrete and App-Based Manipulatives to } \\
\text { Support Students With Disabilities With Adding Fractions }\end{array}$ \\
\hline 11 & $\begin{array}{l}\text { Ayesha W;Samina A. e } \\
\text { Shaista M. }\end{array}$ & 2018 & $\begin{array}{l}\text { Development of Number Concepts in Students with Intellectual } \\
\text { Disability by using Digital Game based Learning }\end{array}$ \\
\hline 12 & $\begin{array}{l}\text { Souza, A.C de e Silva, G. } \\
\text { H.da. }\end{array}$ & 2019 & $\begin{array}{l}\text { Incluir não é Apenas Socializar: as Contribuições das Tecnologias } \\
\text { Digitais Educacionais para a Aprendizagem Matemática de Estudantes } \\
\text { com Transtorno do Espectro Autista }\end{array}$ \\
\hline 13 & Bouck, E.C et.al. & 2019 & $\begin{array}{l}\text { Using the Virtual-Abstract Instructional Sequence to Support } \\
\text { Acquisition of Algebra }\end{array}$ \\
\hline
\end{tabular}




\begin{tabular}{|c|l|l|l|}
\hline 14 & Jimenez, B.A. e Besaw, J. & 2020 & $\begin{array}{l}\text { Building early numeracy through virtual manipulatives for students } \\
\text { with intellectual disability and autismo }\end{array}$ \\
\hline 15 & Bouck, E.C et.al. & 2020 & $\begin{array}{l}\text { Learning Fractions with a Virtual Manipulative Based Graduated } \\
\text { Instructional Sequence }\end{array}$ \\
\hline $\mathbf{1 6}$ & $\begin{array}{l}\text { Triwahyuningtyas, D. et.al } \\
\text { Teaching basic mathematics and technology to elementary students } \\
\text { with autismo }\end{array}$ \\
\hline $\mathbf{1 8}$ & $\begin{array}{l}\text { Yakubova, G; Hughes, } \\
\text { E.M. e Chen, B.B. }\end{array}$ & 2020 & $\begin{array}{l}\text { Neaching students with ASD to solve fraction computations using a } \\
\text { video modeling instructional package }\end{array}$ \\
\hline $\mathbf{1 9}$ & $\begin{array}{l}\text { Bouck, E.C; Park, J e } \\
\text { Shurr, J. }\end{array}$ & 2021 & $\begin{array}{l}\text { Development of a Mobile App to Improve Numeracy Skills of } \\
\text { Children with Autism Spectrum Disorder: Participatory Design and } \\
\text { Usability Study }\end{array}$ \\
\hline $\mathbf{2 0}$ & $\begin{array}{l}\text { Park, J. and Bouck, E.C e } \\
\text { Fisher, M. H }\end{array}$ & 2021 & $\begin{array}{l}\text { Using the virtual-representational instructional sequence to support the } \\
\text { acquisition and maintenance of mathematics for students with } \\
\text { intellectual disability }\end{array}$ \\
\hline $\mathbf{2 1}$ & $\begin{array}{l}\text { Shurr, J. Bouck, E.C.; } \\
\text { Bassette, L. e Park, J. }\end{array}$ & 2021 & $\begin{array}{l}\text { Using the Virtual-Representational-Abstract With Overlearning } \\
\text { Instructional Sequence to Students With Disabilities in Mathematics }\end{array}$ \\
\hline
\end{tabular}

3. Resultados e Discussões Após a leitura de todos os trabalhos, foi realizado um agrupamento, a fim de analisar como se concentram os resultados em relação ao públicoalvo das pesquisas, visto que essa RSL aborda o uso de A.P e tecnologias para apoiar o ensino de matemática para sujeitos com TEA e D.I. Os agrupamentos deram-se da seguinte forma:

- Pesquisas que referem apenas sujeitos com TEA: (Id) 2,3,5,7,12,17,18,21.

- Pesquisas que referem apenas sujeitos com D.I: (Id) 1,6,10,11,13,19.

- Pesquisas que referem sujeitos com D.I e TEA: (Id) 4,8,9,14,15,16,20.

A seguir, o Gráfico1 ilustra os percentuais de grupos de sujeitos encontrados nesta RSL:

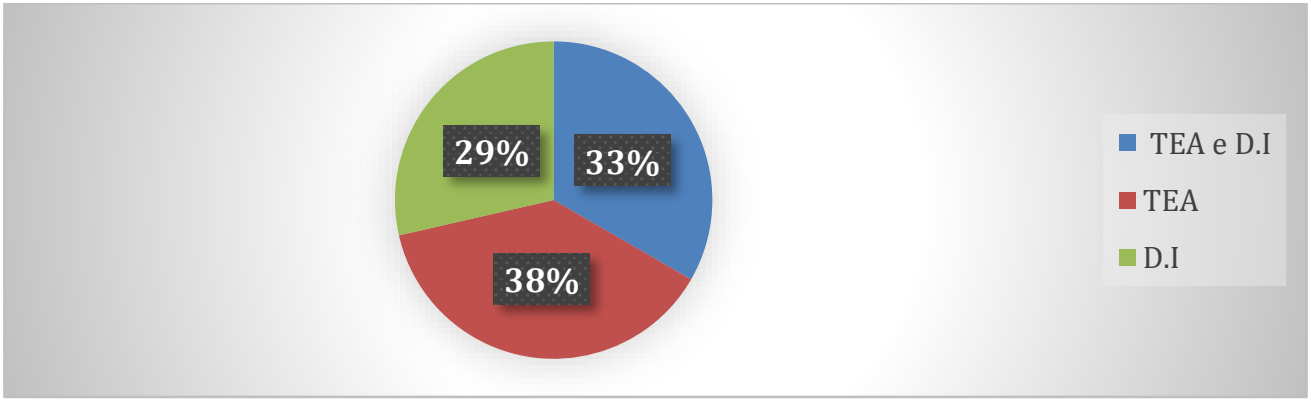

Figura 2: Agrupamento do Público-alvo das pesquisas

É possível observar que dentre as pesquisas para apoiar o ensino de matemática a educandos com TEA e/ou D.I, os resultados apresentam uma média de 33\% que relacionam as pesquisas a essas duas deficiências. Observa-se que muitos trabalhos (ver se tiver porcentagem) relatam que os sujeitos possuíam os dois diagnósticos concomitantemente.

\subsection{QP1: Quais as A.P ou tecnologias que estão sendo usadas para apoiar o ensino de matemática a educandos com TEA e/ou D.I?}

Ao analisar os 21 trabalhos com relação aos tipos de tecnologias digitais ou A.P que vêm sendo usadas para apoiar o ensino de matemática a educandos com TEA e/ou D.I, constatou-se que 11 trabalhos utilizaram em suas propostas aplicativos para mobile, como 
celulares, smartphone, tabletes e ipad, estes são apresentados na seção 2 pelos indicadores 4,5,9,10,13,14,15,18,19,20,21. Alguns dos aplicativos citados são: Splash Math, Motiom Zom e long (id.4); Proyecta @matematicas (id.5); Keymath independente, e Fraction Tiles (id.10, 15); Álgebra Tiles (i.d 13); Azulejos cor (id.20); Base Tens Blocks (id. 21).

Os trabalhos que utilizavam como tecnologias ambientes inteligentes virtuais foram 4, sendo indicados pelos id. 1, 2, 7 e 8. Sendo que, estes são ambientes produzidos pelos próprios pesquisadores ou adaptados para o uso em suas pesquisas. Destacamos a pesquisa de Sula et.al (2014) que apresenta um ambiente assistivo inteligente com sensores e leitor de tag, e Santos et.al (2017) propõem um ambiente desenvolvido com atividades denominado LEMA, o qual os sujeitos podem interagir e receber diferentes feedbacks.

Quatro (4) dos resultados apresentavam a utilização de jogos virtuais como recursos, são eles: id. 6, 11,12 e 16. Dentre as propostas de jogos, a grande maioria trata-se de relacionar o objeto a sua quantidade, ou marcar as opções corretas de perguntas a sua resposta, onde cada sujeito vai avançando de nível à medida que vai acertando os resultados, assim, o grau de dificuldade também aumenta, à medida que o educando consegue avançar as etapas do jogo.

Houve um trabalho que utilizou como recurso o software Geogebra, com atividades interativas e significativas (id.3) e um estudo que utilizou como recurso uma proposta de modelagem com vídeos, onde os sujeitos assistiam vídeos clipes, e depois praticavam e refletiam sobre suas compreensões individuais e tentavam solucionar as atividades apoiados por materiais concretos (id. 17). Destacamos que não foi encontrada nenhuma pesquisa que apresentasse o uso de uma A. P para apoiar o ensino de matemática ou álgebra à educandos com TEA e/ou D.I.

A seguir, a figura 2 ilustra os tipos de tecnologias utilizadas em cada pesquisa:

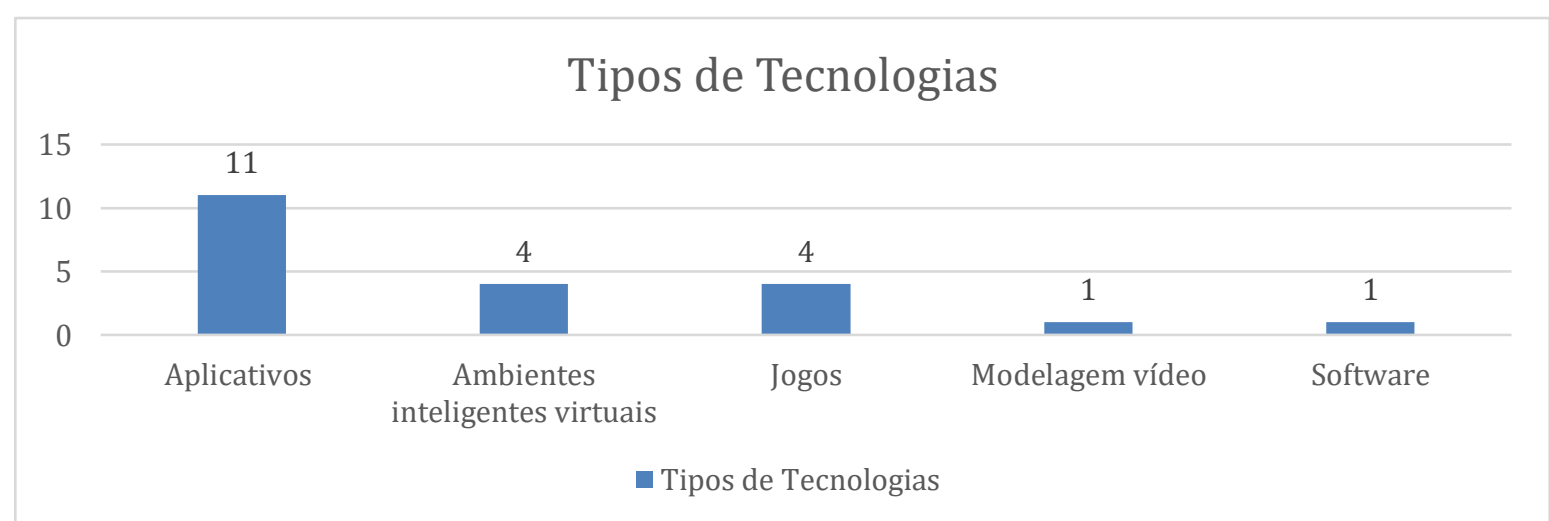

Figura 3: Tipos de tecnologias utilizadas em cada pesquisa

\section{2 QP2: Existem pesquisas sobre o uso A.P ou tecnologias para apoiar o ensino específico de álgebra à educandos com TEA e/ou D.I?}

Referente aos achados sobre o ensino de álgebra apoiado por tecnologias para sujeitos com TEA e/ou D.I, foi localizada apenas 1 trabalho, onde Bouck et.al (2019) descrevem a utilização do aplicativo Álgebra Tiles para um grupo de quatro alunos do ensino médio, diagnosticados com D.I, para verificar a viabilidade do uso de uma sequência de instrução virtual para ensinar álgebra linear. Os autores relatam que a tecnologia utilizada foi um meio eficaz para ensinar os estudantes a adquirir comportamentos matemáticos, que todos os quatro participantes da pesquisa atingiram com sucesso três comportamentos de álgebra linear dentro de seis a sete sessões de instrução, cada uma com menos de 20 minutos. 
Os demais trabalhos localizados nas buscas, abordavam em grande parte o uso de tecnologias para apoiar o ensino das quatro operações básicas (adição, subtração, multiplicação e divisão), e para aprendizagens de operações com frações para sujeitos dos anos iniciais do ensino fundamental.

\subsection{QP3: O uso de tecnologias digitais no ensino matemática com educandos com TEA e/ou D.I é realizado de maneira cooperativa entre os sujeitos?}

Ao analisar as pesquisas com uso de A.P ou tecnologias para apoiar o ensino matemática a sujeitos com TEA e D.I, umas das questões de pesquisa foi compreender se essas práticas permitiam a cooperação entre os educandos, visto que uma das características de uma A.P é a aprendizagem cooperativa.

Dentre os 21 trabalhos lidos, apenas a proposta de Bakker et.al (2016), (id. 6) utiliza uma abordagem pedagógica interativa e cooperativa entre os sujeitos, de forma que os sujeitos jogavam inicialmente de forma individual, e após realizavam uma prática em grupo, de forma que cada sujeito apresenta suas estratégias e discutiam quais são mais favoráveis de se aplicar no jogo, em outro momento, os educandos têm a oportunidade de jogar novamente, assim os sujeitos são levados a pensar, discutir e refletir sobre as melhores estratégias, ou seja, uma prática que favorece as tomadas de consciência de cada sujeito.

As demais pesquisas analisadas usaram tecnologias desenvolvidas para o sujeito interagir de forma individual em ambientes virtuais, jogos, softwares e aplicativos e não propuseram nenhum momento de diálogo ou reflexão, para os sujeitos, de suas práticas.

\subsection{QP4: As propostas pedagógicas no ensino de matemática com o uso de A.P ou tecnologias digitais para educandos com TEA e/ou D.I baseiam-se em uma abordagem construtivista?}

Nesta Q.P foram analisadas se as tecnologias são utilizadas a partir de uma abordagem construtivista, ou seja, que permita o sujeito, ao interagir com o meio (objeto de conhecimento), passe por um processo de conceitualização, através da reflexão sobre sua ação, e, consequentemente, tenha tomada de consciência (PIAGET, 1977). Foram analisados os trabalhos que propiciavam os sujeitos serem os protagonistas no processo de construção de conhecimento (PIAGET, 2002).

Os artigos identificados pelos indicadores 1,3, 6, 7, 9, 10, 11, 12, 13, 14, 15, 17, 18, 19, 20, e 21 apresentaram práticas pedagógicas com os sujeitos, de forma que, indicam uma abordagem construtivista, mesmo que a metodologia dessas pesquisas não apontasse, foi possível concluir que, esses, propunham práticas onde o sujeito interagia com o objeto de conhecimento, visando novas aprendizagens.

No trabalho de Reis et.al (2010) (id.1), por exemplo, é apresentado um conjunto de exercícios multimídia que permite o sujeito interagir com a internet, além de ferramentas semióticas, símbolos, sinais e sons, que transformam esse ambiente em uma proposta utilizada a serviço do processo de construção do conhecimento. Em Root et.al, a proposta construtivista apresenta-se quando os autores propõem que os educandos trabalhem com manipuladores virtuais e um organizador gráfico, assim, o sujeito interage através da manipulação e exploração.

É importante destacar que os demais trabalhos (id. 2,4,5,8 e 16) não deixaram claro as propostas pedagógicas, ou, no caso do objeto de aprendizagem apresentado por MunozSoto (2016) (id. 5), os autores descrevem como sendo um ambiente padronizado para o 
sujeito reproduzir os exercícios de adição, subtração, figuras geométricas e sistema monetário. Assim, a mera reprodução de práticas ou exercícios não proporciona os desequilíbrios necessários para que possa refletir sobre sua prática ou ação, sendo apenas mais um processo mecânico de realizar as atividades.

\section{Considerações Finais}

Esta pesquisa apresentou os resultados de uma RSL realizada para verificar quais A.P e tecnologias vêm sendo utilizadas com educandos com TEA e D.I para apoiar o ensino de matemática. Foram analisados 21 artigos e seus dados foram extraídos para responder as questões de pesquisa.

Durante as buscas, foi possível constatar que o número de pesquisas sobre o uso de tecnologias vem crescendo. Mas, ao referirmos o uso de tecnologias no ensino de matemática e a inclusão do público-alvo da educação especial, como sujeitos com TEA e D.I, ou mesmo pesquisas que analisem as tecnologias em contextos que tenham incluídos esses sujeitos, os resultados são incipientes, e, dentro do universo de 322 trabalhos, menos de 10\% (apenas 21) abordam essa temática. Ao olharmos mais especificamente para abordagens sobre o ensino de álgebra mediadas por ferramentas tecnológicas a este público, os resultados foram escassos, e foi localizado apenas 1 trabalho.

Foi possível concluir que a maioria dos estudos apresenta abordagens de aprendizagens construtivista, demonstrando que as propostas pedagógicas de ensino de matemática apoiadas por tecnologias têm sido um suporte essencial para auxiliar nas atividades, e, dessa forma, auxiliando no processo de pensar, refletir e, assim, criarem novos conhecimentos, a partir de sucessivos desequilíbrios, pois são nessas interações (do sujeito com objeto) que os sujeitos terão a tomada de consciência de um esquema de ação e, consequentemente, uma conceitualização (PIAGET, 1977, 2002).

Por fim, destacamos que não foi localizado nenhuma pesquisa sobre o uso de A.P para apoiar as aprendizagens de matemática para educandos com TEA e/ou D.I. Como perspectiva de trabalhos futuros, acreditamos que o desenvolvimento de uma A.P para apoiar o ensino de álgebra elementar para estes sujeitos possa ser de grande importância, e demonstra ser um trabalho inovador no contexto do uso de A.P aplicadas a aprendizagens matemáticas e ao pensamento algébrico aos sujeitos público-alvo da educação especial.

\section{Referências}

AMERICAN PSYCHIATRIC ASSOCIATION - APA. Diagnostic and Statistical Manual of Mental Disorders ( $5^{\mathrm{a}}$ ed.) Arlington, VA: American Psychiatric Publishing, 2013.

ARAGÓN, R. Interação e mediação no contexto das arquiteturas pedagógicas para a aprendizagem em rede. Cuiabá. Revista Educação Pública. V.25, n 59, p. 261-275. Maio/agosto 2016.

BOSA, C. A. Autismo: atuais interpretações para antigas observações. In C. R. Baptista \& C. A. Bosa (Orgs.), Autismo e educação: reflexões e propostas de intervenção (pp. 21-39). Porto Alegre: Artmed. 2002.

BOUCK, E; PARK, J; SATSANGI, R; CWIAKALA, K; LEVY, R. Using the VirtualAbstract Instructional Sequence to Support Acquisition of Algebra. 20019. Diponível em: https://doi.org/10.1177/0162643419833022 
BURTON, C; ANDERSON, D; PRATER, M; DYCHES, T. Video self modeling on an ipad to teach functional math skills to adolescents with autism and intellectual disability. Focus on Autism and Other Developmental Disabilities. Disponível em: http://foa.sagepub.com/content/early/2013/03/04/1088357613478829.abstract.Bakker.2013.

CHEN, W. (2012). Multitouch tabletop technology for people with autism spectrum disorder: A review of the literature. Procedia Computer Science, 14(1877), 198-207. https://doi.org/10.1016/j.procs.2012.10.023.

DIAS, A. M. A inclusão de alunos com Transtorno do Espectro Autista (Síndrome de Asperger): Uma proposta para o ensino de química. Dissertação de mestrado. UFPEL. Pelotas, 2017.

INHELDER, B. Le diagnostic du raisonnement chez les débiles mentaux. Delachaux et Niestlé. 1963.

KITCHENHAM, B. (2004) "Procedures for Performing Systematic Reviews", Technical Report TR/SE-0401. Department of Computer Science. Disponível em: https://www.inf.ufsc.br/ aldo.vw/kitchenham.pdf .

KITCHENHAM, B. and Charters, S. (2007). Guidelines for performing systematic literature reviews in software engineering. Disponível em: https://www.elsevier.com/_data/promis_misc/525444systematicreviewsguide.pdf.

MENEZES, C. S.; NEVADO, R. A.; ZIEDE, M. L.; CHARCZUK, S. B. Arquiteturas Pedagógicas para a Aprendizagem em Rede no Contexto do Seminário Integrador. RENOTE - Revista Novas Tecnologias na Educação, vol. 11, n. 02, 2013.

MUNOZ-SOTO, R, et al. Proyect@ Matematicas: A Learning Object for Supporting the Practitioners in Autism Spectrum Disorders. IEEE, p 131. 2016.

NEVADO, R. A. de; MENEZES, C. S. de; VIEIRA JUNIOR, R. R. Debate de teses-uma arquitetura pedagógica. In: SIMPÓSIO BRASILEIRO DE INFORMÁTICA NA EDUCAÇÃO, 23., 2011, Aracaju. Anais [...]. Aracaju: SBIE. p. 820-829.

PIAGET, J. A Tomada de Consciência. Com a colaboração de A. Blamchet [e outros]. Tradução de Edson B. de Souza. São Paulo: Melhoramentos, 1977.

. Epistemologia Genética. 4 ed. São Paulo: Martins Fontes, 2002.

PONTE, J. P. da; BRANCO, N.; MATOS, A. álgebra no Ensino Básico. Ministério da Educação de Portugal, 2009.

REIS, M. et al. Using Information Technology Based Exercises in Primary Mathematics Teaching of Children with Cerebral Palsy and Mental Retardation: A Case Study. TOJET the Turkish online journal of educational technology. 2010.

SANTOS, M; BREDA, A; ALMEIDA, A. Design approach of mathematics learning activities in a digital environment for children with autism spectrum disorders. 2017. Disponível em: https://doi.org/10.1007/s11423-017-9525-2.

SULA. A; SPAHO, E; BAROLLI, L; MIRO, R. A Proposed framework for combining smart environment and heuristic diagnostic teaching principles in order to assess students' abilities in math and supporting them during learning. 2014. 